\title{
Some Graceful Graphs
}

\author{
V. J. Kaneria (Corresponding author) \\ Department of Mathematics, Saurashtra University, Rajkot 360005, Gujarat, India \\ E-mail: kaneria_vinodray_j@yahoo.co.in
}

H. M. Makadia

Government Engineering College, Rajkot 360005, Gujarat, India

E-mail: makadia.hardik@yahoo.com

Received: September 13, 2011 Accepted: October 8, 2011 Published: February 1, 2012

doi:10.5539/jmr.v4n1p54 URL: http://dx.doi.org/10.5539/jmr.v4n1p54

\begin{abstract}
We have investigated some general classes of disconnected graceful graphs. We obtain the graceful labeling for $\left(P_{m} \times\right.$ $\left.P_{n}\right) \cup\left(P_{r} \times P_{s}\right), C_{2 f+3} \cup\left(P_{m} \times P_{n}\right) \cup\left(P_{r} \times P_{s}\right)$, where $m, n, r, s \in N-\{1\}$ and $f=2(m n+r s)-(m+n+r+s)$. We also show that the tensor product of $P_{n}$ and $P_{3}$, where $n \in N-\{1,2\}$ admits graceful labeling. In addition to this we prove that a graph called star of cycle $C_{n}^{\star}$ is graceful, for $n \equiv 0(\bmod 4)$.
\end{abstract}

Keywords: Path graph, Grid graph, Graceful graph, Star of cycle

AMS Subject classification number(2010): $05 \mathrm{C} 78$

\section{Introduction}

We begin with simple, undirected and finite graph $G=(V(G), E(G))$ with $p$ vertices and $q$ edges. In this work $C_{n}$ denotes the cycle on $n$ vertices, $P_{n}$ denotes the path on $n$ vertices and $\left(P_{n} \times P_{m}\right)$ denotes the grid graph on $m n$ vertices. For all other terminology and notations we follow (West, 2001).

1.1 Definition If the vertices are assigned values subject to certain conditions then it is known as graph labeling.

1.2 Definition A function $f$ is called graceful labeling of a graph $G=(V(G), E(G))$ if $f: V(G) \longrightarrow\{0,1,2, \ldots, q\}$ is injective and the induce function $f^{*}: E(G) \longrightarrow\{1,2, \ldots, q\}$ defined as $f^{*}(e=u v)=|f(u)-f(v)|$ is bijective, $\forall e=u v$ $\in E(G)$. A graph which admits graceful labeling is called graceful graph.

Rosa introduced this concept in (Rosa, 1967, p. 349-355) and named it as $\beta$-valuation. Golomb (Golomb, 1972 , p. 23-37) discovered such labeling independently and called it graceful labeling which is now the popular term. The famous graceful tree conjecture and several attempts to settle it provided the reason for extensive research in the field of graph labeling. Acharya (Acharya, 1982, p. 231-236) constructed certain infinite families of graceful graphs from a given graceful graph. Acharya and Hegde (Acharya \& Hegde, 1990, p. 275-299) generalized graceful labeling to $(k, d)$ graceful labeling while Acharya and Singh (Acharya \& Singh, 2004, p. 291-302) have discussed gracefulness of signed graphs. Moreover Rosa(Rosa, 1967, p. 349-355) and Golomb (Golomb, 1972, p. 23-37) discussed gracefulness of complete graphs and Eulerian graphs. For a dynamic survey on graph labeling we refer to (Gallian, 2010).

1.3 Definition The Cartesian product of graphs $G$ and $H$ denoted as $G \times H$, is the graph with vertex set $V(G) \times V(H)=$ $\{(u, v) / u \in V(G)$ and $v \in V(H)\}$ and $(u, v)$ adjacent to $\left(u^{\prime}, v^{\prime}\right)$ if and only if either $u=u^{\prime}$ and $v v^{\prime} \in E(H)$ or $v=v^{\prime}$ and $u u^{\prime} \in E(G)$.

1.4 Definition The Cartesian product of two paths $P_{n}$ and $P_{m}$ denoted as $\left(P_{n} \times P_{m}\right)$ is known as a grid graph on mn vertices.

1.5 Definition The tensor product of two graphs $G_{1}$ and $G_{2}$ denoted by $G_{1}\left(T_{p}\right) G_{2}$ has vertex set $V\left(G_{1}\left(T_{p}\right) G_{2}\right)=V\left(G_{1}\right) \times$ $V\left(G_{2}\right)$ and the edge set $E\left(G_{1}\left(T_{p}\right) G_{2}\right)=\left\{\left(\left(u_{1}, v_{1}\right),\left(u_{2}, v_{2}\right)\right) / u_{1} u_{2} \in E\left(G_{1}\right)\right.$ and $\left.v_{1} v_{2} \in E\left(G_{2}\right)\right\}$.

In (Bu \& Cao, 1995, p. 6-8) have discussed gracefulness of complete bipartite graph and its union with path. In (Acharya \& Gill, 1981, p. 81-94) have investigated the graceful labeling for the grid graph $\left(P_{n} \times P_{m}\right)$. In this paper we have discussed gracefulness of $\left(P_{m} \times P_{n}\right) \cup\left(P_{r} \times P_{s}\right), C_{2 f+3} \cup\left(P_{m} \times P_{n}\right) \cup\left(P_{r} \times P_{s}\right)($ with $m, n, r, s \in N-\{1\}$ and $f=2(m n+r s)-(m+n+r+s))$, $P_{n}\left(T_{p}\right) P_{3}($ with $n \in N-\{1,2\})$ and $C_{n}^{\star}($ with $n \equiv 0(\bmod 4))$. 


\section{Gracefulness of Some Product Related Graphs}

2.1 Theorem The graph $G=\left(P_{n} \times P_{m}\right) \cup\left(P_{r} \times P_{s}\right)$, where $m, n, r, s \in N-\{1\}$ is graceful.

Proof: It is obvious that the graph $G$ has number of vertices $p=r s+m n$ and number of edges $q=2(r s+m n)-(m+n+r+s)$.

Now label the vertices of $P_{n} \times P_{m}$ by the labels $q, 0,1, q-2, q-3, q-4,4,5, \ldots$ etc to diagonals of the grid $P_{n} \times P_{m}$. This labeling sequence is having two sequential patterns, one is increasing and the other is decreasing. Such labeling will give rise to edge labeling as decreasing sequence of labels $q, q-1, \ldots, q+m+n+1-2 m n$. The described labeling pattern demonstrated in Figure-1.

Now our task is to label the vertices of $P_{r} \times P_{s}$. It will depend on the vertex labels of the last grid of $P_{n} \times P_{m}$. Let $w$ and $t$ be vertex labels of last grid of $P_{n} \times P_{m}$. These labels produce edge label $q+m+n+1-2 m n=2 r s+1-(r+s)$. At this stage we have to consider following two cases.

Case - I: $w<t$. Then $w$ must be a label from the increasing sequence of labels and $t-w=2 r s+1-(r+s)$. Now the available vertex labels are $t+1, t-1, t-2, \ldots, w+2, w+1$, which are in number $2 r s+1-(r+s)$.

We will use these labels for labeling of vertices $P_{r} \times P_{s}$. This vertex labeling sequence is $2 r s-(r+s)+w+2, w+2, w+$ $3,2 r s-(r+s)+w, 2 r s-(r+s)+w-1,2 r s-(r+s)+w-2, w+7, w+8, \ldots$ etc. This labeling sequence will give rise to edge labels as $2 r s-(r+s), \ldots, 2,1$. Thus we have labeled all the $r s+m n$ vertices of $G$ gracefully.

Case - II: $w>t$. Then $w$ must be a label from the decreasing sequence of labels and $w-t=2 r s+1-(r+s)$. Now the available vertex labels are $w-1, w-2, \ldots, t+2, t+1, t-1$, which are in number $2 r s+1-(r+s)$.

We will use these labels for labeling of vertices of $P_{r} \times P_{s}$, in which vertex labeling sequence is $t-1, w-2, w-3, t+1, t+2$, $t+3, w-7, \ldots$ etc. This will give rise to edge labels as decreasing sequence of labels $2 r s-(r+s), \ldots, 2,1$. Thus we have labeled all the $r s+m n$ vertices of $G$ gracefully.

Therefore $G=\left(P_{n} \times P_{m}\right) \cup\left(P_{r} \times P_{s}\right)$ is a graceful graph.

2.2 Illustration Graceful labeling of $\left(P_{3} \times P_{4}\right) \cup\left(P_{4} \times P_{2}\right)$ is shown in Figure - 2 .

2.3 Theorem The graph $G=C_{2 f+3} \cup\left(P_{m} \times P_{n}\right) \cup\left(P_{r} \times P_{s}\right)$, where $m, n, r, s \in N-\{1\}$ and $f=2(m n+r s)-(m+n+r+s)$ is graceful.

Proof: It is obvious that $G$ will have number of vertices $p=2 f+3+m n+r s$ and number of edges $q=3 f+3$. Let $u_{1}, u_{2}, \ldots, u_{2 f+3}$ be the successive vertices of $C_{2 f+3}$.

Define the vertex labeling function $f: V(G) \longrightarrow\{0,1,2, \ldots, q\}$ for the cycle $C_{2 f+3}$ as

$f\left(u_{i}\right)=(q+1)-\frac{i}{2}=(3 f+4)-\frac{i}{2}$; if $i$ is even

$=\frac{i-1}{2}$; if $i$ is odd.

Which will produce edge labels $3 f+3,3 f+2, \ldots, f+2, f+1$ for the cycle $C_{2 f+3}$. Now our task is to label the vertices of $\left(P_{m} \times P_{n}\right) \cup\left(P_{r} \times P_{s}\right)$ for which the available vertex labels are $2 f+2,2 f+1, \ldots, f+2$ and which may give rise to edge labels $f, f-1, \ldots \ldots, 2,1$.

Since the number of available vertex labels are $f+1$ and required number of edge labels are $f$. For that we first label the vertices of $\left(P_{m} \times P_{n}\right) \cup\left(P_{r} \times P_{s}\right)$ by $0,1, \ldots, f$, as suggested in Theorem-2.1 and then add the number $f+2$ to all the vertex labels of $\left(P_{m} \times P_{n}\right) \cup\left(P_{r} \times P_{s}\right)$ in order to produce edge labels $1,2, \ldots, f$ for $\left(P_{m} \times P_{n}\right) \cup\left(P_{r} \times P_{s}\right)$.

Thus we have labeled the graph $G=C_{2 f+3} \cup\left(P_{m} \times P_{n}\right) \cup\left(P_{r} \times P_{s}\right)$ gracefully. Therefore $G$ is a graceful graph.

\section{Gracefulness of $P_{n}\left(T_{p}\right) P_{3}$ and Star of Cycle}

3.1 Theorem $P_{n}\left(T_{p}\right) P_{3}$, the tensor product of $P_{n}$ and $P_{3}$, where $n \in N-\{1,2\}$ is a graceful graph

Proof: Let $v_{i j}$ be successive vertices of $P_{n}\left(T_{p}\right) P_{3}, \forall i=1,2,3$ and $\forall j=1,2, \ldots, n$. Now define the labeling function $f: V\left(P_{n}\left(T_{p}\right) P_{3}\right) \longrightarrow\{0,1, \ldots, q=4 n-4\}$ as follows.

$f\left(v_{i j}\right)=j-1+\frac{i-1}{2}$; if $j$ is odd

$$
=2\left\lceil\frac{n+1}{2}\right\rceil-1+j+\frac{i-1}{2} \text {; if } j \text { is even, } \forall i=1,3 .
$$

and $f\left(v_{2 j}\right)=q+6-2\left\lceil\frac{n}{2}\right\rceil-j$; if $j$ is odd

$$
=q+2-j \text {; if } j \text { is even. }
$$

Such vertex labeling for $P_{n}\left(T_{p}\right) P_{3}$ will produce edge labels $q, q-1, \ldots, \frac{q}{2}+1$ and $\frac{q}{2}, \frac{q}{2}-1, \ldots, 2,1$. Thus $f$ is a graceful 
labeling function and consequently $P_{n}\left(T_{p}\right) P_{3}$ is a graceful graph.

3.2 Illustration: The graph $P_{5}\left(T_{p}\right) P_{3}$ and its graceful labeling is shown in Figure - 3 .

3.3 Definition A graph obtained by replacing each vertex of $\operatorname{star} K_{1, n}$ by a graph $G$ of $n$ vertices is called star of $G$ and it is denoted by $G^{\star}$. The graph $G$ which replaced at the center of $K_{1, n}$ we call the central copy of $G^{\star}$.

Above definition was introduced in (Vaidya et al, 2008, p. 54-64).

3.4 Theorem $C_{n}^{\star}$, the star of cycle $C_{n}$ is graceful graph, when $n \equiv 0(\bmod 4)$.

Proof: Let $v_{1}, v_{2}, \ldots, v_{n}$ be successive vertices of central copy of $C_{n}^{\star}$ and $u_{i 1}, u_{i 2}, \ldots, u_{i n}$ be successive vertices of cycles $C_{n}^{(i)}, i=1,2, \ldots, n$. Let $e_{i}$ be edge such that $e_{i}=v_{i} u_{i-1 i}, \forall i=2,3, \ldots, n$ and $e_{1}=v_{1} u_{n 1}$, which join each cycle $C_{n}^{(i)}$ with the central copy of $C_{n}^{\star}$. Now we define the labeling function $f: V\left(C_{n}^{\star}\right) \longrightarrow\{0,1, \ldots, q=n(n+2)\}$ as follows:

$$
\begin{aligned}
f\left(u_{1 i}\right) & =\frac{n^{2}+q}{4}+\frac{i-1}{2} \text { if } i \equiv 1(\bmod 2) \\
& =\frac{n^{2}+q}{4}+n+1-\frac{i}{2} \text { if } i \equiv 0(\bmod 2) \text { and } i \leq \frac{n}{2} \\
& =\frac{n^{2}+q}{4}+n-\frac{i}{2} \text { if } i \equiv 0(\bmod 2) \text { and } i>\frac{n}{2}
\end{aligned}
$$

Case - I : $j \equiv 1(\bmod 2)$ and $3 \leq j \leq n-1$.

$$
\begin{aligned}
f\left(u_{j i}\right) & =f\left(u_{(j-1) i}\right)-2(j-1)(n+1) \text { if } i \equiv 0(\bmod 2) \\
& =f\left(u_{(j-1) i}\right)+2(j-1)(n+1) \text { if } i \equiv 1(\bmod 2)
\end{aligned}
$$

Case - II : $j \equiv 0(\bmod 2)$ and $2 \leq j \leq n$.

$$
\begin{aligned}
& \qquad \begin{aligned}
f\left(u_{j i}\right) & =f\left(u_{(j-1) i}\right)+2(j-1)(n+1) \text { if } i \equiv 0(\bmod 2) \\
& =f\left(u_{(j-1) i}\right)-2(j-1)(n+1) \text { if } i \equiv 1(\bmod 2)
\end{aligned} \\
& \text { and } f\left(v_{i}\right)=f\left(u_{1 i}\right)+\frac{n^{2}+q}{4} \text { if } i \equiv 0(\bmod 2) \\
& =f\left(u_{(1 i}\right)-\frac{n^{2}+q}{4} \text { if } i \equiv 1(\bmod 2) .
\end{aligned}
$$

Such vertex labeling for $C_{n}^{\star}$ will produce edge labels $(i-1)(n+1)+1,(i-1)(n+1)+2,(i-1)(n+1)+3, \ldots, i(n+1)$ for the cycles $C_{n}^{(i)}, i=1,2, \ldots, n$ and edge labels $n(n+1)+1, n(n+1)+2, \ldots, n(n+2)$ for the central cycle. Also it will produce edge labels $(n+1), 2(n+1), \ldots, n(n+1)$ for edges $e_{i}$, which join each cycle $C_{n}^{(i)}$ with the central copy of $C_{n}^{\star}$. Thus we have labeled all the vertices of $C_{n}^{\star}$ gracefully. That is, $C_{n}^{\star}$ is a graceful graph.

3.5 Illustration The graph $C_{4}^{\star}$ and its graceful labeling is shown in Figure-4.

\section{Concluding Remarks}

Here we have discussed the gracefulness of the grid graph and its union with some other graphs. We have also derived that the tensor product of $P_{n}$ and $P_{3}$ admits graceful labeling. The results obtained here are new and of very general nature. This work throws some light on the gracefulness of disconnected graphs which is a very less explored field.

\section{Acknowledgement}

The authors are highly thankful to the anonymous referees for their constructive suggestions and kind comments.

\section{References}

Acharya B. D., \& Gill M. K. (1981). On the index of gracefulness of a graph and the gracefulness of two-dimensional square lattice graphs, Indian J. Math., 23, 81-94.

Acharya B. D. (1982). Construction of Certain infinite families of graceful graphs from a given graceful graph. Defence Sci. J., 32, 231-236.

Acharya B. D., \& Hegde S. M. (1990). Arithmetic graphs, J. Graph Theory, 14(3), 275-299.

Acharya M., \& Singh T. (2004). Czech. Math J., 54(129), 291-302.

Bu C., \& Cao C. (1995). The gracefulness for a class of disconnected graphs, J. Natural Sci. Heilon gjiang Uni., 12 , 6-8. Gallian J. A. (2010). A dynamic survey of graph labeling, The Electronic Journal of Combinatorics, $17, \sharp \mathrm{DS} 6$.

Golomb S. W. (1972). How to number a graph. In: Graph Theory and Computing (R. C. Read. Ed.) Academic Press. New York, 23-37. 
Rosa A. (1967). On certain valuations the vertices of a graph, Theory of Graphs (Rome, July 1966), Gordon and Breach, N. Y. and Dunod Paris, 349-355.

Vaidya S. K., Srivastav S., Kaneria V. J., \& Ghodasara G. V. (2008). Cordial and 3-equitable labeling of star of a cycle, Mathematics Today, 24 , 54-64.

West D. B. (2001). Inroduction to Graph Theory, Prentice-Hall of India, New Delhi.

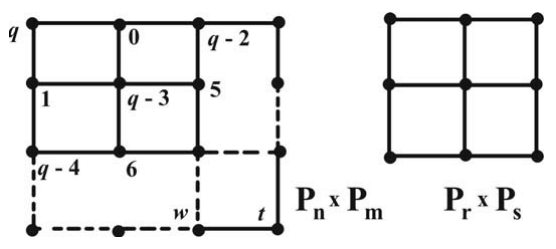

Figure-1
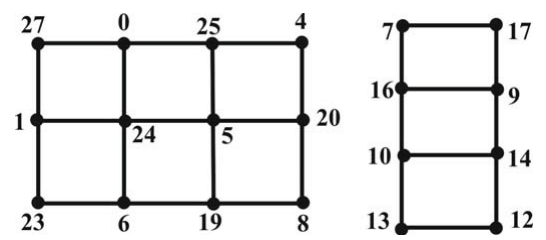

Figure-2

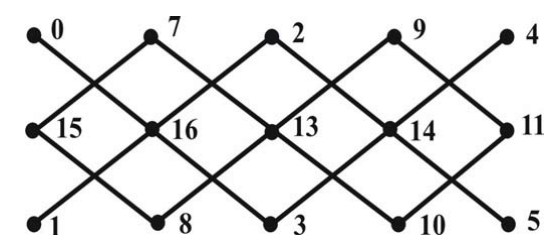

Figure-3

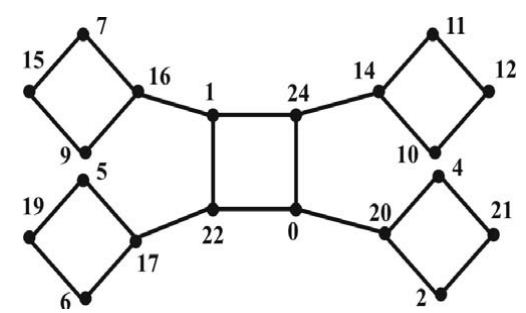

Figure-4

Figure $1-4$ 